\title{
Chemical Structure, Hypoglycemic Activity, and Mechanism of Action of Selenium Polysaccharides
}

\author{
Wen-Xia Duan ${ }^{1} \cdot$ Xiao-Hua Yang ${ }^{2} \cdot$ Hua-Feng Zhang ${ }^{1,3} \cdot$ Jing Feng ${ }^{4} \cdot$ Meng-Yuan Zhang ${ }^{1,3}$
}

Received: 14 September 2021 / Accepted: 14 November 2021 / Published online: 29 November 2021

( ) The Author(s), under exclusive licence to Springer Science+Business Media, LLC, part of Springer Nature 2021

\begin{abstract}
Selenium polysaccharides (Se-polysaccharides) are one of important forms of organic Se, in which selenium (Se) and polysaccharides are joined by covalent bonds. In the present review, recent progress in chemical structure and hypoglycemic activity of Se-polysaccharides is summarized. In particular, the mechanism underlying hypoglycemic capacity of Se-polysaccharides is discussed, and the relationship between hypoglycemic activity and chemical structure is analyzed. Besides, strategies for further research into chemical structure and hypoglycemic activity of Se-polysaccharides are proposed. Hypoglycemic activity of Se-polysaccharides is closely related to their inhibitory effect on $\alpha$-amylase and $\alpha$-glucosidase, influence on insulin signal pathway especially IRS-PI3K-Akt signaling pathway, and protection capacity against oxidative stress.
\end{abstract}

Keywords Se-polysaccharides $\cdot$ Chemical structure $\cdot$ Hypoglycemic activity $\cdot$ Mechanism of action $\cdot$ Structure-activity relationship

\section{Introduction}

Selenium (Se) is a crucial trace element possessing distinctive beneficial effects on human health [1]. Se intake seems to have protective effects against influenza, acquired immunodeficiency syndrome (AIDS), and even coronavirus disease 2019 (COVID-19) [2, 3]. And Se deficiency or dyshomeostasis may lead to several epidemics such as Keshan disease, cardiovascular disease, asthma, male sterility,

Hua-Feng Zhang

isaacsau@sohu.com

1 Provincial Research Station of Se-Enriched Foods in Hanyin County of Shaanxi Province, International Joint Research Center of Shaanxi Province for Food and Health Sciences, National Engineering Laboratory for Resources Development of Endangered Crude Drugs in Northwest China, Shaanxi Normal University, Xi' an 710062, People's Republic of China

2 Health Science Center, Xi' an Jiaotong University, Xi' an 710061, People's Republic of China

3 Municipal Expert Workstation for Hua-Feng Zhang, Academician and Expert Workstation in Pu'er City of Yunnan Province, Pu'er 665600, People's Republic of China

4 Agrarian and Technological Institute, Peoples' Friendship University of Russia, Moscow 119991, Russia cancers, and diabetes mellitus $[4,5]$. In nature, Se exists in two states: inorganic and organic forms. When compared with inorganic Se, organic Se frequently exhibits superior advantages such as lower toxicity, fewer side effects, and higher bioavailability [4]. Usually, Se intake must depend on Se supplements (e.g., selenocysteine), Se-containing foods, or Se-enriched foods (e.g., Se-enriched Chrysanthemum morifolium) [5]. Selenium polysaccharides (Se-polysaccharides), an important form of organic Se, are widely used as Se supplements [6]. Se-polysaccharides are actually a Se complex composed of Se and polysaccharides. It is known that polysaccharides display a broad range of bioactivities such as antihyperlipidemic, immune-regulatory, antioxidant, antitumor, and antiaging capacities [7, 8]. Currently, increasing studies demonstrated that Se-polysaccharides showed higher bioactivities than single polysaccharides or Se, especially in terms of hypoglycemic activity [9].

Type 2 diabetes mellitus (T2DM) is a complicated clinical syndrome with disorders of carbohydrate and lipid metabolisms usually accompanied by several complications [10]. Generally, insulin resistance (IR) is considered the fundamental pathogenesis of T2DM, which is mainly caused by impaired insulin signaling pathways and other multiple metabolic pathways such as lipid oxidation, protein synthesis and degradation, and hepatic gluconeogenesis [11]. When IR happens, response of peripheral tissues (e.g., liver, muscle 
and fat cells) to insulin stimulation most probably downregulates, which eventually results in metabolic disorder of blood glucose [12]. If human is exposed to the overnourished state generated by IR for a long time, insulin will not effectively inhibit liver gluconeogenesis and stimulate glucose intake of peripheral tissues while insulin-stimulated lipolysis remains intact [13]. Over the past 20 years, many papers that dealt with hypoglycemic capacities of Se-enriched vegetables, fruits, and medicinal plants have been published [13-16]. Our previous research revealed that aqueous and methanolic extracts from Se-enriched Chrysanthemum morifolium possessed stronger antioxidant capacities as well as inhibitory activities against $\alpha$-amylase and $\alpha$-glucosidase in comparison with traditional Chrysanthemum morifolium (non-Seenriched Chrysanthemum morifolium). Furthermore, Sepolysaccharides isolated from Se-enriched Chrysanthemum morifolium markedly reduce postprandial blood glucose and remit the typical symptoms such as polydipsia and polyphagia in diabetic mice $[15,16]$. It is of theoretical and applied significance to investigate chemical structure, hypoglycemic activity, and its mechanism of Se-polysaccharides and exploit novel phytopharmaceuticals or nutraceuticals based upon Se-polysaccharides for prevention and treatment of T2DM.

In this review, we shall provide an overview of recent progress in chemical structure and hypoglycemic activity of Se-polysaccharides. In particular, we shall discuss structure-activity relationship of Se-polysaccharides. Besides, the mechanism underlying hypoglycemic capacity of Sepolysaccharides will be analyzed. Comprehensive understanding of mechanism of action and structure-activity relationship of Se-polysaccharides may enable the discovery of novel nutraceuticals or pharmaceuticals targeting insulin signaling pathways, which is helpful to restore glucose homeostasis and ameliorate hyperglycemia. At last, we shall propose strategies to unveil the mechanism underlying hypoglycemic activity of Se-polysaccharides and to explore their clinical application to T2DM.

\section{Techniques for Preparing Natural and Synthetic Se-Polysaccharides}

\section{Natural Se-Enriched Polysaccharides}

\section{Se-Polysaccharides from Plants}

Plants are one of the main sources of natural Se-polysaccharides (Table 1), which frequently possess health benefits to human [6]. Usually, the formation of Se-polysaccharides in plants depends on concentration, transport, and anabolism of Se from soils to plants [17]. And transport of Se in plants is carried out via delivery systems for other elements
[18], because there is no specific delivery systems for Se. El Mehdawi et al. [19] revealed that Se transport and accumulation in Stanleya pinnata is intimately linked with overexpression of sulfate transporters SULTR1 and SULTR2. Similarly, Zhang et al. [20] also indicated that overexpression of phosphorus transporter OsPT2 was involved in Se accumulation in rice. Unfortunately, absorption and transport of Se can be hampered by competitive ions such as sulfate and phosphate anions in soils [21]. Furthermore, Se forms in soils greatly affected absorption and transport of Se. Se in soils can be divided into the following forms: (1) soluble Se, (2) exchangeable and carbonate-bound Se, (3) iron $(\mathrm{Fe})$-manganese $(\mathrm{Mn})$ oxide-bound $\mathrm{Se},(4)$ organic matter-bound Se, and (5) the residual state [22]. Among them, only soluble Se and exchangeable Se are regarded as available Se in soils, which can be assimilated by plants [23]. In our laboratory, Se-enriched matcha has been manufactured based on a specific variety of Camellia sinensis cultivated in the famous selenium-enriched region, Ziyang County, Ankang City, Shaanxi Province of China (Chinese Patent No. ZL202021318642.3). In many case, available Se is less than 5\% in soils, which partially contributed to low content of Se-polysaccharides in plants [21]. According to the WHO, about 1 billion people are suffering from Se malnutrition due to low Se level in soils, which leads to insufficiency of Se in crops [24]. In order to improve Se content in crops, some techniques were employed during their cultivation and production. On one hand, inorganic Se was artificially sprayed on aerial parts of crops (Fig. 1). On the other hand, Se-rich fertilizers were put on soils.

\section{Se-Polysaccharides from Microorganisms}

When microorganisms absorbed exogenetic Se oxyanions (e.g., $\mathrm{SeO}_{4}{ }^{2-}$ and $\mathrm{SeO}_{3}{ }^{2-}$ ) or elementary $\mathrm{Se}\left(\mathrm{Se}^{0}\right)$, they manufactured Se-polysaccharides with Se and polysaccharides in cell walls [25]. Zhou et al. [26] successfully prepared microbial Se-polysaccharides by adding $\mathrm{Na}_{2} \mathrm{SeO}_{3}$ to the culture medium of Enterobacter cloacae. Malinowska et al. [27] obtained two kinds of Se-polysaccharides using Hericium erinaceus with $\mathrm{Na}_{2} \mathrm{SeO}_{3}$ and organoselenium (i.e., selenite triglycerides), respectively. Compared to $\mathrm{Na}_{2} \mathrm{SeO}_{3}$, organoselenium contributed to higher yield of Se-polysaccharides and lower toxic effects.

\section{Extraction of Natural Se-Polysaccharides}

Currently, separation and purification methods of Se-polysaccharide are similar to conventional polysaccharides (non-Se-polysaccharides). And water decoction and ethanol precipitation method is the most common technique for extracting Se-polysaccharides from biological matrix. The method usually exhibited the following advantages: (1) low 
Table 1 Chemical property and character of natural and synthetic Se-polysaccharides

\begin{tabular}{|c|c|c|c|c|c|c|}
\hline Original source & Se-polysaccharides & Preparation method & $\begin{array}{l}\text { Methods for } \\
\text { Se detection }\end{array}$ & Se content $(\mu \mathrm{g} / \mathrm{g})$ & $\begin{array}{l}\text { Monosaccharide composi- } \\
\text { tion }\end{array}$ & References \\
\hline \multirow[t]{3}{*}{ Oudemansiella radicata } & SPS & \multirow[t]{3}{*}{ Bio-transformation } & \multirow[t]{3}{*}{ FAAS } & 127.10 & Rib, Man, Gal, Glu & \multirow[t]{3}{*}[6]{} \\
\hline & ESPS & & & 93.10 & Man, Gal, Glu & \\
\hline & ASPS & & & 83.20 & Rha, Fuc, Glu, Man, Gal & \\
\hline Momordica charantia & Se-MCPIIa-1 & $\mathrm{Vc} / \mathrm{Na}_{2} \mathrm{SeO}_{3}$ & AFS & 445 & $\begin{array}{l}\text { Man, Fuc, Ara, Rha, Gla, } \\
\text { Gaa, Glu, Gal, Xyl }\end{array}$ & [7] \\
\hline \multirow[t]{2}{*}{ Green tea } & CSe-TPS & \multirow[t]{2}{*}{ NA-SS } & \multirow[t]{2}{*}{ AFS } & 2120 & $\begin{array}{l}\text { Rha, Ara, Gluc, Gal, Glu, } \\
\text { Xyl, Man, Fru, Gaa }\end{array}$ & \multirow[t]{2}{*}[8]{} \\
\hline & ASe-TPS & & & 1640 & $\begin{array}{l}\text { Rha, Ara, Gal, Glu, Xyl, } \\
\text { Man, Fru, Gaa }\end{array}$ & \\
\hline \multirow[t]{2}{*}{ Green tea } & SeTPS-1 & \multirow[t]{2}{*}{ Bio-transformation } & \multirow[t]{2}{*}{ AFS } & 23.50 & Glu, Gal & \multirow[t]{2}{*}{ [54] } \\
\hline & SeTPS-2 & & & 13.47 & Ara, Glu, Gal, Gaa & \\
\hline Tea & Se-TPS & Bio-transformation & AFS & 1.987 & $\begin{array}{l}\text { Fuc, Rha, Ara, Gal, Glu, } \\
\text { Man, Rib, Gla, Gaa }\end{array}$ & {$[50]$} \\
\hline Pyracantha fortuneana & Se-PFPs & Bio-transformation & - & - & - & [9] \\
\hline Murus alba & MFP3P-Se & NA-SS & AAS & 452 & Rha, Ara, Gal, Glu, Gaa & [12] \\
\hline Enterobacter cloacae & Se-ECZ-EPS & $\mathrm{Se}^{0} \mathrm{NPs}$ & - & - & - & [26] \\
\hline \multirow[t]{2}{*}{ Hericium erinaceum } & EPS & \multirow[t]{2}{*}{ Bio-transformation } & \multirow[t]{2}{*}{ AAS } & 4890 & Man, Glu, Gal & \multirow[t]{2}{*}{ [27] } \\
\hline & IPS & & & 4690 & Glu, Gal, Man & \\
\hline Castanea mollissima & sCPA & NA-SS & ICP-OES & 573.9 & Glu & [29] \\
\hline Pleurotus geesteranus & SPMP-2a & Bio-transformation & - & - & - & {$[31]$} \\
\hline Artemisia sphaerocephala & $\mathrm{SeASP}_{\mathrm{MW}}$ & NA-SA & AAS & $111 \sim 264$ & Ara, Xyl, Glu, Man, Gal & {$[32]$} \\
\hline Glycyrrhiza uralensis & Se-GUP & NA-SS & ICP-OES & 1339 & - & [33] \\
\hline Schisandra chinensis & SCPs & NA-SS & AFS & $3810 \sim 10,350$ & - & {$[34]$} \\
\hline Artemisia sphaerocephala & Se-ASP & $\mathrm{SeOCl}_{2}$ method & AFS & 22,400 & - & {$[35]$} \\
\hline \multirow[t]{4}{*}{ Garlic } & \multirow[t]{4}{*}{ GPSs } & NA-SS & \multirow[t]{4}{*}{ AFS } & 29,400 & \multirow[t]{4}{*}{-} & \multirow[t]{4}{*}{ [39] } \\
\hline & & GA-SA & & 26,300 & & \\
\hline & & GA-SS & & 10,500 & & \\
\hline & & $\mathrm{SeOCl}_{2}$ method & & 9200 & & \\
\hline Capparis spinosa & Se-CSPS & NA-SS & UV & $2438 \sim 5547$ & - & [40] \\
\hline Potentilla anserina & SePAP & NA-SA & HPLC & $2690.1 \pm 7.2$ & Glu, Man, Gal & [41] \\
\hline Sweet potato & Se-SWP & NA-SS & AFS & 12,740 & Rha, Glu, Gal & {$[42]$} \\
\hline Cordyceps sinensis & EPS-SeNPs & $\mathrm{Se}^{0} \mathrm{NPs}$ method & - & - & - & [45] \\
\hline Undaria pinnatifida & Se-polysaccharides & $\mathrm{Se}^{0} \mathrm{NPs}$ method & - & - & - & [46] \\
\hline Platycodon grandiflorum & PGP1 & Bio-transformation & AFS & 39.4 & Glu, Gal, Man & {$[53]$} \\
\hline Grifola frondosa & Se-GFP-22 & Bio-transformation & AFS & 8.37 & Man, Glu, Gal & {$[55]$} \\
\hline Catathelasma ventricosum & SemCVP-1Ss & NA-SS & ICP-MS & $1180 \sim 1860$ & Glu, Gal, Fuc & {$[52]$} \\
\hline Alfalfa & Se-RAPS-2 & NA-SS & AFS & 320 & $\begin{array}{l}\text { Rha, Xyl, Gaa, Man, Glu, } \\
\text { Gal }\end{array}$ & {$[57]$} \\
\hline Catathelasma ventricosum & SPC-2 & Bio-transformation & AFS & 41.77 & Glu, Xyl, Man, Gal & {$[51]$} \\
\hline Catathelasma ventricosum & CVPs-SeNPs & $\mathrm{Se}^{0} \mathrm{NPs}$ method & - & - & - & {$[58]$} \\
\hline Cordyceps sinensis & ISPS & Bio-transformation & - & - & - & [59] \\
\hline \multirow[t]{3}{*}{ Cordyceps militaris } & SeCPS-I & \multirow[t]{3}{*}{ NA-SS } & \multirow[t]{3}{*}{ AFS } & 541.3 & \multirow[t]{3}{*}{ Man, Glu, Gal } & \multirow[t]{3}{*}[60]{} \\
\hline & SeCPS-II & & & 863.7 & & \\
\hline & SeCPS-II & & & 623.3 & & \\
\hline
\end{tabular}

$N A-S S$ nitric acid-sodium selenite, $S e^{0} N P s$ elementary Se with nanometer size, $N A-S A$ nitric acid-selenous acid, $G A-S S$ glacial acetic acidsodium selenite, $G A-S A$ glacial acetic acid-selenous acid, $F A A S$ flame atomic absorption spectrometry, $A F S$ atomic fluorescence spectrometer, $A A S$ atomic absorption spectrometer, ICP-OES inductively coupled plasma-optical emission spectrometer, $U V$ UV-Vis spectrophotometer, ICP$M S$ inductively coupled plasma mass spectrophotometer, HPLC high-performance liquid chromatography, Rha rhamnose, Ara arabinose, Gluc glucosamine, Glu glucose, Gal galactose, Xyl xylose, Man mannose, Fru fructose, Gaa galacturonic acid, Gla glucuronic acid, Fuc fucose, Rib ribose 
Fig. 1 Bioactivities of natural and synthetic Se-polysaccharides

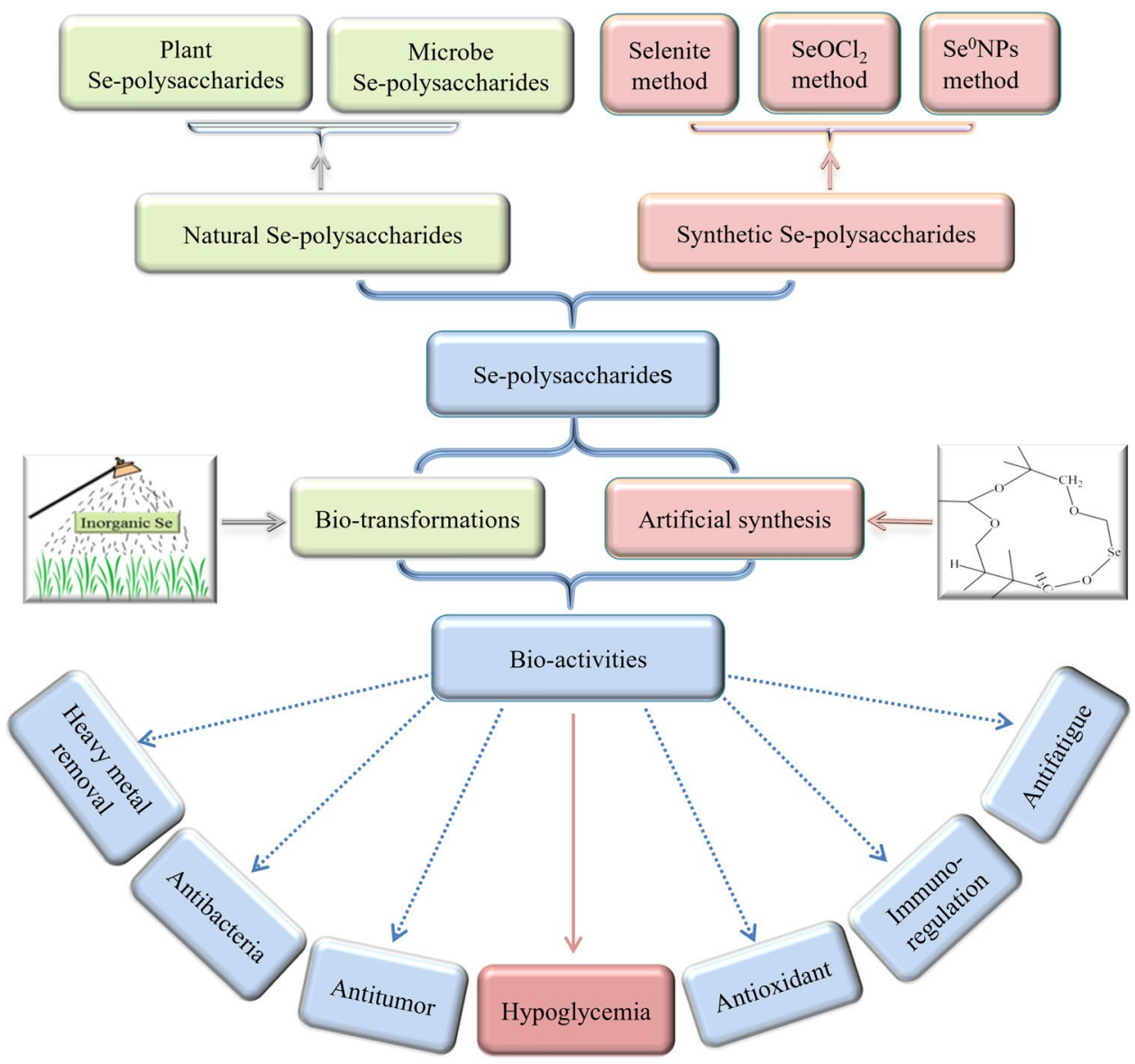

solvent consumption, (2) low cost, and (3) operating simplicity $[28,29]$. Ultrasonic extraction was also used to isolate Se-polysaccharides from Se-enriched Lyophyllum decastes. However, too long ultrasonication time probably caused degradation of Se-polysaccharides. One possible reason is that acoustic cavitation produced by ultrasound may rupture the surface of polysaccharides. Another reason is that ultrasound may produce free radicals, which probably damage the structure of polysaccharides [30].

\section{Artificially Synthetic Se-Polysaccharides}

Since abundance of Se in plants and other organisms is usually low, Se intake from natural crops or foods cannot satisfy people's physical needs in many cases, which is called "hidden hunger of Se." Concerns about Se-polysaccharides deficiency in natural organisms and hidden hunger of Se in human populations have fostered investigations into artificial synthesis of Se-polysaccharides [31]. Presently, artificial synthesis of Se-polysaccharides with evident health benefits has attracted more attention (Table 1). Polysaccharides are macromolecules with a vast array of chemical groups such as hydroxyl, aldehyde, ketone, and amino groups, some of which can be replaced by selenide reagent and then form Se-polysaccharides.

In general, there are three common ways of chemically synthesizing Se-polysaccharides. The first method is selenylation modification of polysaccharides with selenide reagents [32]. Elementary Se, sodium selenite, and selenous acid are usually employed as selenide reagents modifying chemical structure of target polysaccharides. And dominant systems of selenylation modification include nitric acid-sodium selenite (NA-SS), nitric acid-selenous acid (NA-SA), glacial acetic acid-sodium selenite (GA-SS), and glacial acetic acid-selenous acid (GA-SA) [14]. Wang et al. [32] achieved a series of Se-polysaccharides with Se content of $168-1703 \mu \mathrm{g} / \mathrm{g}$ using polysaccharides extracted from Artemisia sphaerocephala as the substrate (precursor) and $\mathrm{BaCl}_{2}$ as the catalyst by NA-SA system. And they found that $\mathrm{Ba}^{2+}$ notably improves selenylation efficiency of polysaccharides. By the similar system, Lian et al. [33] also synthesized Se-polysaccharides with Se content of $1339 \mu \mathrm{g} / \mathrm{g}$ based on Glycyrrhiza uralensis polysaccharides, which exhibited significant antioxidant activities in vivo and in vitro. Likewise, NA-SS system was applied to preparation of Se-enriched Schisandra chinensis polysaccharides [34]. The second method is selenylation modification of polysaccharides with 
selenium oxychloride $\left(\mathrm{SeOCl}_{2}\right)$. $\mathrm{SeOCl}_{2}$, one of active acylation agents, has strong capacity to conjugate with hydroxyl groups of polysaccharides [35]. Chemically synthetic Sepolysaccharides with hypoglycemic activity were achieved based upon Artemisia sphaerocephala polysaccharides using $\mathrm{SeOCl}_{2}$ method [35]. Most recently, selenized glucose with in vivo antioxidant activity was synthesized, which might be used as an alternative precursor for de novo synthesis of Se-polysaccharides [36-38].

In general, Se yield in end-products of Se-polysaccharides is considered a crucial parameter of selenylation modification [39]. As regards the first method (selenite method), Se yields by NA-SA system $(0.91-0.11 \%)$ and GA-SA system $(0.73-0.50 \%)$ are higher than that of the second method ( $\mathrm{SeOCl}_{2}$ method) (0.33-0.04\%) in many cases. Moreover, its operation is simpler than the second method [39], although it is more time-consuming. For instance, selenylation of glucan from Castanea mollissima fruits with Se content of $574 \mu \mathrm{g} / \mathrm{g}$ was performed using NA-SS system at $65^{\circ} \mathrm{C}$ for $10 \mathrm{~h}$ [29]. Likely, selenylation of polysaccharides from Capparis spinosa with Se content of $2438-5545 \mu \mathrm{g} / \mathrm{g}$ was conducted using HA-SS system at $65^{\circ} \mathrm{C}$ for $10 \mathrm{~h} \mathrm{[40].} \mathrm{It} \mathrm{is}$ noticeable that long heating duration may cause degradation of polysaccharides in presence of $\mathrm{HNO}_{3}$. As regards the second method ( $\mathrm{SeOCl}_{2}$ method), toxic gas is usually released in the reaction process [39]. Reaction time of Se-enriched Artemisia sphaerocephala polysaccharides obtained by $\mathrm{SeOCl}_{2}$ method $(5 \mathrm{~h})$ was remarkably lower than that by NA-NS system $(16 \mathrm{~h})[32,35]$. To shorten reaction time of selenylation, microwave technique was employed to assist selenite method and $\mathrm{SeOCl}_{2}$ method [41, 42]. It only took $2 \mathrm{~h}$ to prepare Se-enriched Potentilla anserina polysaccharides with high Se yield of $2687.24 \mu \mathrm{g} / \mathrm{g}$ by NA-SA system with assistance of microwave irradiation [41]. Similarly, Seenriched Ipomoea batatas polysaccharides with Se content of $12.74 \mathrm{mg} / \mathrm{g}$ were synthesized at $100{ }^{\circ} \mathrm{C}$ for $0.5-0.8 \mathrm{~h}$ by NA-SA system [42]. Microwave is a kind of electromagnetic wave with high frequency, which can be converted into thermal energy and rapidly heat the reactants when passed through the reactive medium [43]. Microwave exhibited remarkable ability to shorten reaction time and increase $\mathrm{Se}$ content during Se-polysaccharide synthesis.

Interestingly, Se nanoparticles (elementary Se with nanometer size, $\mathrm{Se}^{0} \mathrm{NPs}$ ) were used to artificially synthesize Se-polysaccharides [44]. For example, Xiao et al. [45] successfully prepared well-dispersed nano-Se-polysaccharides (CVPs-Se ${ }^{0} \mathrm{NPs}$ ) using Lignosus rhinocerotis polysaccharides as surface modifier of $\mathrm{Se}^{0} \mathrm{NPs}$ and ascorbic acid as reducing agent of sodium selenite, respectively. Chen et al. [46] also obtained Se-polysaccharides by adding Undaria pinnatifida polysaccharides into the reaction system of selenite and ascorbic acid. Acute toxicity tests indicated that $\mathrm{LD}_{50}$ (median lethal dose) of $\mathrm{Se}^{0} \mathrm{NPs}(15.70 \mathrm{mg} / \mathrm{kg}$ ) was significantly lower than that of $\mathrm{H}_{2} \mathrm{SeO}_{3}(113.00 \mathrm{mg} / \mathrm{kg})$ [47]. Estevez et al. [48] found that toxicity to HepG2 cell considerably varied among different $\mathrm{Se}$ forms (Se(IV), $\mathrm{Se}(\mathrm{VI})$, and $\mathrm{Se}^{0} \mathrm{NPs}$ ), and $\mathrm{Se}^{0} \mathrm{NPs}$ only caused slight toxicity to HepG2 cells when compared to $\mathrm{Se}(\mathrm{IV})$ and $\mathrm{Se}(\mathrm{VI})$.

\section{Structural Characterization of Se-Polysaccharides}

\section{Methods for Structure Identification of Se-Polysaccharides}

It is already well established that function of biological macromolecules (e.g., polysaccharides) is closely related to their chemical structure [29]. Consequently, it is necessary to thoroughly analyzed chemical structure of Sepolysaccharides by various methods and to reveal their structure-activity relationship. In general, commonly used methods for structural identification of Se-polysaccharides mainly include chemical methods and instrumental ones. As regards chemical methods, carbohydrates and proteins are frequently quantified by phenol- $\mathrm{H}_{2} \mathrm{SO}_{4}$ method and Bradford method, respectively [39]. In our laboratory, a sensitive, precise, and accurate phenol- $\mathrm{H}_{2} \mathrm{SO}_{4}$ method has been developed for determination of polysaccharides in edible medicinal plants [28]. Partial acid hydrolysis, periodate oxidation-Smith degradation and methylation are usually employed to ascertain the position of glycosidic linkages and to analyze the number of branch of polysaccharides [49]. As regards instrument method, molecular weight of Se-polysaccharides is determined by high-performance gel permeation chromatography system, membrane osmometer, and vapor pressure osmometer [50]. Gas chromatography (GC), high-performance liquid chromatography, and mass spectrometry are usually used to identify monosaccharides in Se-polysaccharides [42]. Circular dichroism spectroscopy (CD) is used to characterize secondary structure of Se-polysaccharides. Ultraviolet spectrophotometry (UV), Fourier transform infrared spectroscopy (FT-IR), and nuclear magnetic resonance (NMR) are applied to identification of chemical groups and configurations of glycosidic linkages. Scanning electron microscope (SEM), transmission electron microscope, and atomic force microscope (AFM) are used to observe micromorphology of Se-polysaccharides [51]. In general, Se in Se-polysaccharides is quantified using atomic fluorescence spectrophotometry (AFS), inductively coupled plasma optical emission spectrometer (ICP-OES), and inductively coupled plasma mass spectrometry (ICP-MS) [52]. In our laboratory, a hydride generation atomic fluorescence spectrometric method was developed for quantitative analysis of selenium in plants, which has proved to be an accurate, precise, sensitive, and safe analytical technique [1]. 


\section{Structure of Natural Se-Polysaccharides}

In Se-polysaccharides, Se and polysaccharides are joined by covalent bonds (Fig. 1). Structural analysis of polysaccharides backbone is performed with respect to monosaccharide composition, conformation of glycosidic linkages, binding sites of Se, higher-order structure, as well as micromorphology. GC analysis showed that Se-polysaccharides with Se content of $39.40 \mu \mathrm{g} / \mathrm{g}$ from Se-enriched Platycodon grandiflorum are composed of D-glucose, D-galactose, and D-mannose at molar ratio of 7.1:1.1:0.6 [53]. Sun et al. [31] found that Se-polysaccharides from Se-enriched Pleurotus geesteranus with a molecular weight of $3.32 \times 104 \mathrm{Da}$ belonged to acidic polysaccharides with $\alpha^{-}{ }^{-}$-glucopyranoside bond, and there was a characteristic peak ascribing to $\mathrm{Se}-\mathrm{O}-\mathrm{C}$ stretching vibrations in their infrared spectrum. Structure of two Se-polysaccharides (SeTPS-1 and SeTPS-2) isolated from Se-enriched tea was characterized by GC, FT-IR, Congo red test, and NRM [54]. It was found that SeTPS-1 was composed of glucose and galactose while SeTPS-2 was composed of arabinose, glucose, galactose, and galacturonic acid. Se was attached to polysaccharides backbone in the form of $\mathrm{Se}=\mathrm{O}$ and $\mathrm{O}-\mathrm{Se}-\mathrm{O}$, and two Se-polysaccharides were random coil conformations instead of triple-helix structures [54]. Se-enriched heteropolysaccharides with molecular weight of $4.13 \times 10^{6}$ Da were extracted from Seenriched Grifola frondosa [55]. NRM analysis revealed that there is a backbone chain of 1,4- $\alpha$-linked-D-Glcp units and trace amounts of branches of 1,3,6- $\alpha$-linked-D-Manp and 1,4,6- $\beta$-linked-D-Galp. SEM and AFM images illustrated that irregular fiber net structure existed in Se-polysaccharides, which was similar to results of Congo red analysis [55].

\section{Structure of Synthetic Se-Polysaccharides}

As an alternative to natural Se-polysaccharides, synthetic Se-polysaccharides exerted hypoglycemia activity in some cases [52]. When native polysaccharides were chemically modified using selenide reagents (e.g., selenite, selenate, and elementary Se), the main conformation of polysaccharides remained nearly unchanged. In synthetic Se-polysaccharides derived from chitosan, selenite groups were attached to $\mathrm{C}_{2}$ site of amino group and $\mathrm{C}_{6}$ site of hydroxyl group at polysaccharide chain while the main conformation of polysaccharides remained intact [56]. Congo red test implied that synthetic Se-polysaccharides based on Catathelasma ventricosum mycelial polysaccharides have a triple-helical structure [52]. Raman spectroscopy analysis suggested that Se was attached to polysaccharides backbone in the form of $\mathrm{Se}=\mathrm{O}$ and $\mathrm{C}-\mathrm{O}-\mathrm{Se}$ in synthetic $\mathrm{Se}$-polysaccharides derived from Artemisia sphaerocephala using NA-SA system [32]. Micromorphology of synthetic Se-polysaccharides based upon Medicago sativa roots polysaccharides by NA-SS system was observed using SEM. And they existed in a loose flaky structure, which was different from non-Se-polysaccharides with dense stripped structure [57].

\section{Hyperglycemic Activity and Its Mechanism of Se-Polysaccharides}

\section{Mitigating Activity of Se-Polysaccharides Against Diabetes Mellitus}

T2DM is a chronic disease accompanied with metabolic syndrome and endocrine system disorder. Most recently, increasing incidence of T2DM has seriously threatened to human health. Some reports indicated that Se-polysaccharides possessed remarkable mitigating activity against T2DM with low adverse effects and economic costs (Table 2). In other words, the fact that some Se-polysaccharides could alleviate T2DM provided a rational basis for their medicinal and nutritional uses in future [13, 32]. For instance, Se derivatives of polysaccharides from Ipomoea batatas exhibited mitigating effect on STZ-induced T2DM mice [42]. They could effectively increase body weight, protect organs (kidney, heart, liver, and pancreas) and decrease blood glucose level as well as increase insulin level. In particular, they were not found have lethal effect on mice when they were used as feeds for 30 days [42]. Se-polysaccharides from Catathelasma ventricosum could significantly protect the liver, kidney, and pancreas of STZ-induced T2DM mice, as well as enhance insulin secretion and glycogen synthesis [51].

Numerous studies suggested that hypoglycemic activity was intimately associated with antioxidant activity, especially free radical-scavenging capacity and activating effects on antioxidant enzymes such as superoxide dismutase (SOD) and glutathione peroxidase (GSH-Px) [58]. It is known that SOD plays a protective role in body against oxidative stress especially lipid peroxidation caused by reactive oxygen species (ROS), and GSH-Px mainly reduces $\mathrm{H}_{2} \mathrm{O}_{2}$ to $\mathrm{H}_{2} \mathrm{O}$ to attenuate its oxidative damage. Se-polysaccharides from Cordyceps sinensis could improve SOD and GSH-Px activities in vivo [59]. Se-polysaccharides from Cordyceps militaris could scavenge 1,1-diphenyl-2-picrylhydrazyl (DPPH) radicals, superoxide radicals, and hydroxyl radicals [60]. Likewise, Se-polysaccharides based on Catathelasma ventricosum polysaccharides prepared by $\mathrm{Se}^{0} \mathrm{NPs}$ method could decrease blood glucose, total cholesterol, triglyceride (TG), and low-density lipoprotein cholesterol (LDL-C) and MDA content and improve body weight, high-density lipoprotein cholesterol (HDL-C) level, and activities of antioxidant enzymes such as SOD, catalase (CAT), and GSH-Px in STZ-induced T2DM mice [58]. 


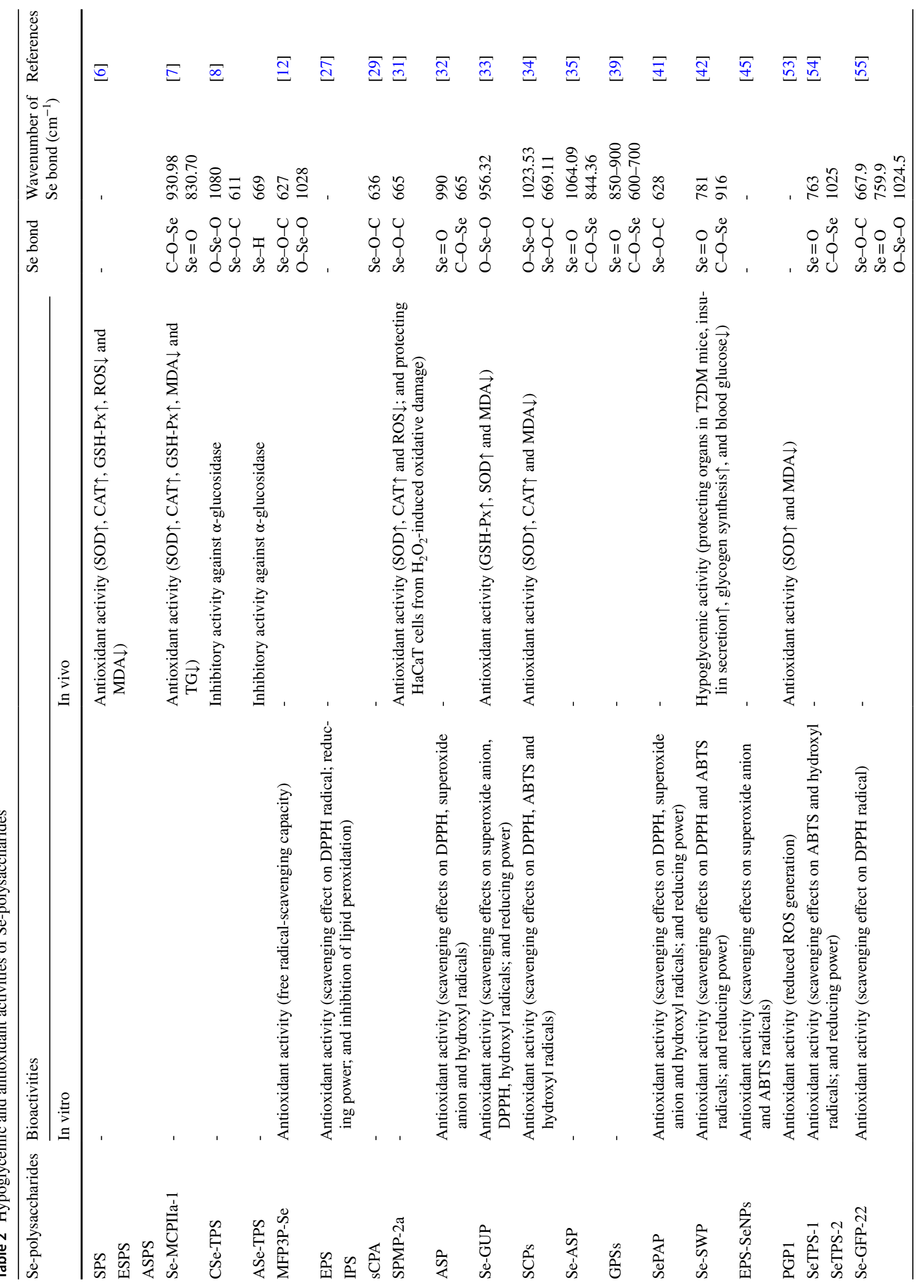




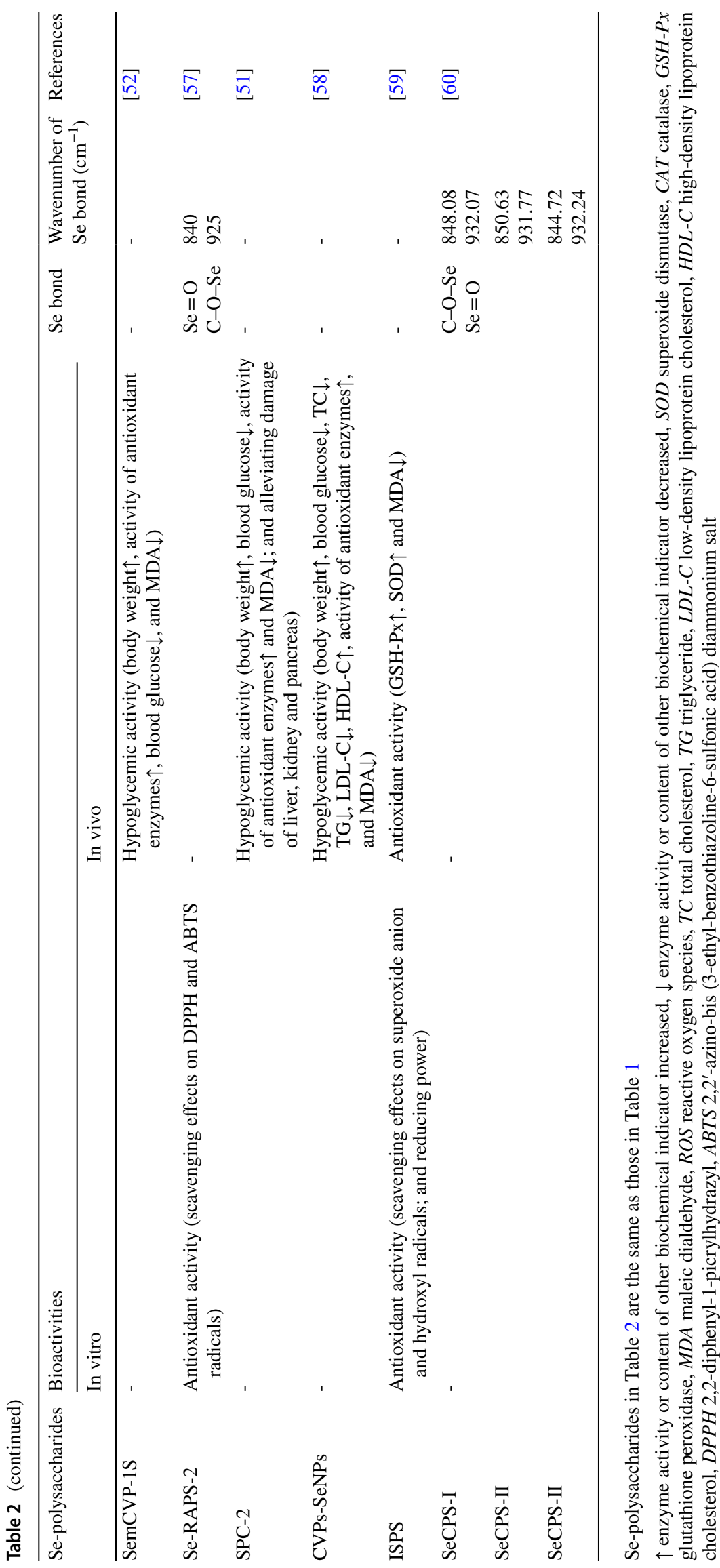




\section{Advantages of Se-Polysaccharides over Other Cures Against Diabetes Mellitus}

With the worldwide epidemic of T2DM, urgent measures should be taken to decrease its morbidity and mortality. Currently, a myriad of chemically synthesized drugs have been widely used to cure or intervene T2DM [61]. Among them, insulin sensitizer (e.g., thiazolidinediones and biguanides), insulin release promoters (e.g., sulfonylureas), and $\alpha$-glucosidase inhibitors (e.g., acarbose and voglibose) were the commonly used oral hypoglycemic agents in clinic [61]. In general, thiazolidinediones (e.g., pioglitazone and rosiglitazone) and biguanides (e.g., metformin) could enhance the sensitivity of insulin receptors in peripheral target tissues, such as the muscle, fat, and liver tissue, ameliorate insulin resistance, inhibit hepatic glucose production, and attenuate hepatic steatosis that involves AMP-activated protein kinase activation [61]. $\alpha$-glucosidase is a key enzyme in carbohydrates digestion and glycoproteins biosynthesis. $\alpha$-glucosidase inhibitors could decrease the release of glucose in the bloodstream by interfering with intestinal $\alpha$-glucosidase activity [62]. That is to say, they might delay carbohydrates digestion and decrease blood glucose. Oral administration of sulfonylureas (e.g., glibenclamide) can provoke secretion of insulin by stimulating pancreatic $\beta$ cells using blocking ATP-sensitive $\mathrm{K}^{+}$channels. However, sulfonylureas potentiate insulin secretion not in glucose-dependent manner, which sometimes cause episodes of hypoglycemia. Besides, a requirement for sulfonylureas to achieve hypoglycemic activity is $30 \%$ living $\beta$ cells with normal metabolic function in diabetic patients, which limits its usefulness in some cases [63]. Unfortunately, there are a number of liabilities and risks inherent in some of the abovementioned chemically synthesized drugs. In general, adverse effects of those drugs included hypoglycemia and gastrointestinal symptoms such as nausea, diarrhea, and vomiting [61]. Additionally, some of hypoglycemic drugs might cause plasma lactic acidosis and osteoporosis [63].

Exogenous insulin and related analogs, commonly used biosynthetic drugs for type 1 and 2 diabetes mellitus, mainly include rapid acting insulin, short acting insulin, intermediate acting insulin, and long acting insulin [64]. Insulin and related analogs possess superior advantages such as flexibility of duration of action. However, they could not simulate rhythmical secretion of endogenous insulin [65]. And their efficacy was greatly affected by the site of injection in many cases. In particular, they failed to decrease high postprandial blood glucose when were injected at unsuitable sites [65]. Therefore, subcutaneous injection of exogenous insulin and related analogs has to obey the guidance of clinicians [51].

Hypoglycemic activity of Se-polysaccharides was confirmed by diabetic animal models induced by STZ, alloxan, or high-fructose diets [13]. Se-polysaccharides are deemed to the "next-generation Se-supplement" due to their merits: (1) Se-polysaccharides exert hypoglycemic activity and antioxidant capacity especially activating effect on antioxidant enzymes; (2) Se-polysaccharides have low toxicity and few side effects; (3) Se-polysaccharides did not cause hypoglycemia [13]; and (4) Se-polysaccharides were not found have lethal effect on experimental animals [42]. Hence, Se-polysaccharides showed great potential for becoming nutraceuticals and pharmaceuticals with relatively high hypoglycemia activity and safety.

\section{Mechanisms Underlying Hypoglycemic Activity of Se-Polysaccharides}

\section{Inhibitory Effect of Se-Polysaccharides on a-Amylase and a-Glucosidase}

Diabetes mellitus is a complicated syndrome with carbohydrate metabolism disorders. $\alpha$-amylase and $\alpha$-glucosidase are two key enzymes in the digestion of carbohydrates, and they could catalyze hydrolysis of starch into glucose. Among them, $\alpha$-amylase is mainly distributed in saliva while $\alpha$-glucosidase is distributed in brush edge of small intestinal epithelial cells [66, 67]. If their activities were inhibited, fasting blood glucose (FBG) might reduce significantly. Consequently, inhibiting activities of $\alpha$-amylase and $\alpha$-glucosidase have possibility for being novel strategy to eliminate hyperglycemia by modulating endogenous glucose production. Currently, some hypoglycemic medicines targeting for $\alpha$-glucosidase have been applied to the treatment of T2DM [67]. Natural Se-polysaccharides from Se-enriched Cyclocarya paliurus exhibited distinctly inhibitory activities against $\alpha$-glucosidase in a dose-dependent manner [68]. Liu et al. [69] isolated two kinds of Se-polysaccharides from Catathelasma ventricosum growing in artificial medium containing $50 \sim 350 \mu \mathrm{g} / \mathrm{mL} \mathrm{Na}_{2} \mathrm{SeO}_{3}$, both of which showed inhibitory activities against $\alpha$-amylase and $\alpha$-glucosidase. In our laboratory, comparison of hypoglycemic activity was performed between Se-polysaccharides extracted from Se-enriched Chrysanthemum morifolium and non-Se-polysaccharides from traditional Chrysanthemum morifolium. And Se-polysaccharides not only inhibit $\alpha$-amylase and $\alpha$-glucosidase, but also decrease blood glucose level in STZinduced diabetic mice in a dose-dependent manner $[15,16]$.

In general, $\alpha$-amylase contains three domains, namely domain $\mathrm{A}$, domain $\mathrm{B}$, and domain $\mathrm{C}$. Among them, domain $A$ is a central $(\alpha / \beta)_{8}$ TIM-barrel structure consisting of symmetric $\alpha$-helices and $\beta$-strands, and domain $\mathrm{B}$ is a small protruding loop structure. Domain A is located at the $\mathrm{N}$-terminal end of enzyme protein, while domains B and C are located at $\mathrm{C}$-terminus. Moreover, domain $\mathrm{A}$ is the catalytic site of enzyme, domain $\mathrm{B}$ is thought to play a role in the substrate specificity, and domain $\mathrm{C}$ is correlated with 
Fig. 2 Possible drug targets of Se-polysaccharides on insulin signaling pathway. Insulin receptor substrates (InsR), phosphatidylinositol-4,5-bisphosphate $\left(\mathrm{PIP}_{2}\right)$, phosphatidylinositol 3,4,5-trisphosphate ( $\mathrm{PIP}_{3}$ ), phosphoinositide 3-kinase (PI3K), Src homology 2 (SH2), protein kinase B (AKt), 3-phosphoinositide-dependent kinase 1 $\left(\mathrm{PDK}_{1}\right)$, 3-phosphoinositide dependent kinase $2\left(\mathrm{PDK}_{2}\right)$, glucose transporter 4 (Glut-4), glycogen synthase kinase- $3 \beta$ (GSK-3 $\beta$ ), possible drug targets of Se-polysaccharides (PDT)

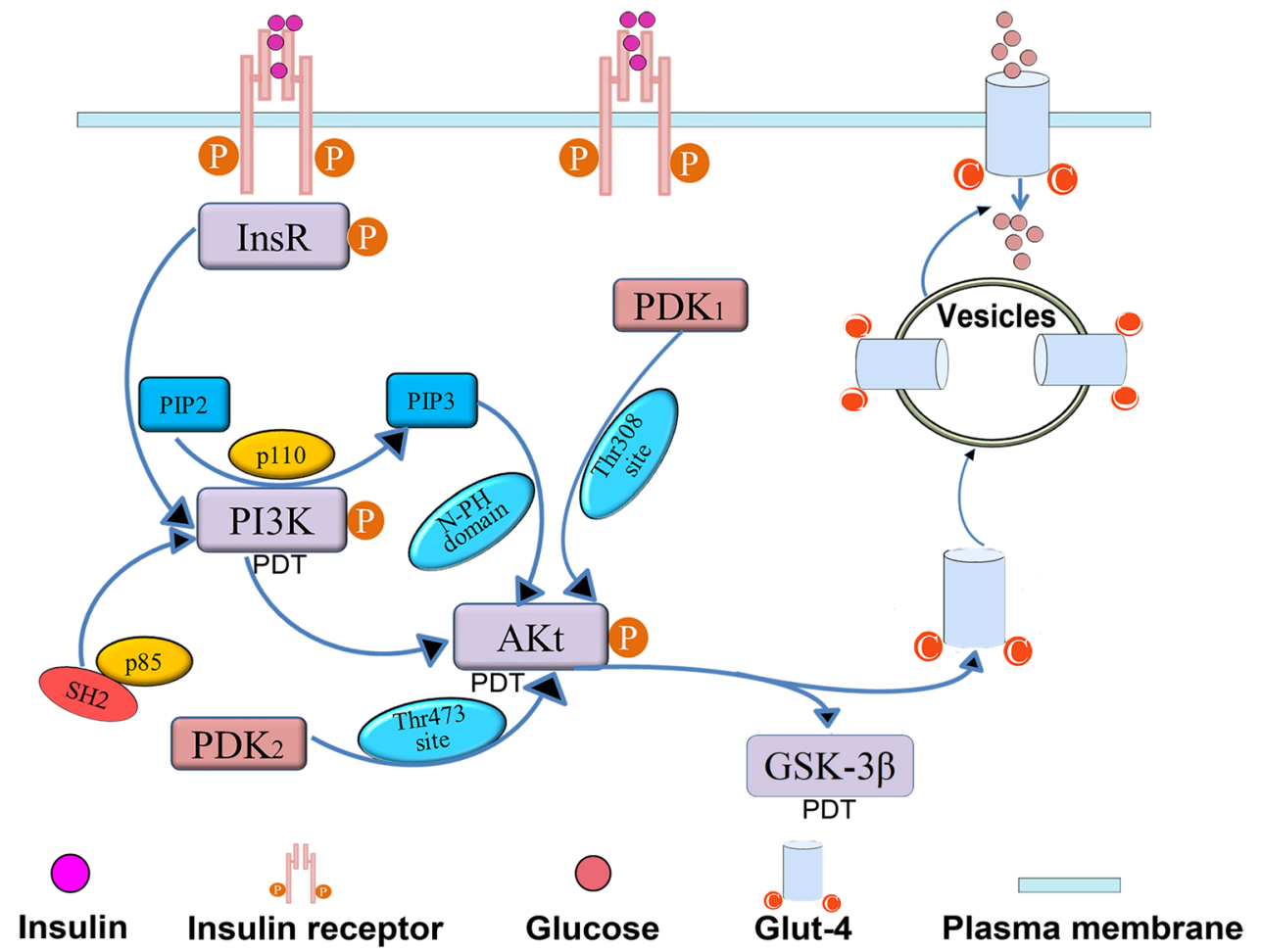

protein stabilization, protein folding and substrate binding [70]. Se-polysaccharides might be competitive inhibitors of $\alpha$-amylases. Unfortunately, there are few reports on hypoglycemic activity of Se-polysaccharides in diabetic animals by inhibiting $\alpha$-amylase and/or $\alpha$-glucosidase. And structural base for inhibition of $\alpha$-amylase and $\alpha$-glucosidase by Sepolysaccharides is still unclear.

\section{Influence of Se-Polysaccharides on Insulin Signal Pathway}

It is known that abnormality of IRS-PI3K-Akt (insulin receptor substrate-phosphoinositide 3-kinase-protein kinase B) signaling pathway is one of main reasons for the episode of insulin resistance [71]. Se-polysaccharides have the potential for improving insulin sensitivity and ameliorating hyperglycemia, which is deemed to be highly correlated with insulin signaling pathway [12]. Indeed, function of insulin is mainly mediated by IRS-PI3K-Akt and MAPK (mitogen-activated protein kinases) insulin signaling pathways [72]. Firstly, insulin receptor is activated when it exclusively combined with insulin. And then, tyrosine kinase in the activated insulin receptor further phosphorylates insulin receptor substrates (InsR). The phosphorylated InsR will bind to $\mathrm{p} 85$ subunits of downstream signaling PI3K and recruit its p110 catalytic subunit to plasma membrane, which leads to the activated PI3K [73]. The high-affinity interaction between $\mathrm{p} 85$ subunits and InsR is realized by $\mathrm{Src}$ homology 2 ( $\mathrm{SH} 2$ ) domain of p85 subunits and phosphorylated tyrosine sequences of InsR [74]. Secondly, activated
PI3K will subsequently phosphorylate its main substrate phosphatidylinositol-4,5-bisphosphate $\left(\mathrm{PIP}_{2}\right)$ and generate the second messenger phosphatidylinositol 3,4,5-trisphosphate $\left(\mathrm{PIP}_{3}\right)$ (Fig. 2). $\mathrm{PIP}_{3}$ is recruited to bind to the pleckstrin homology $(\mathrm{PH})$ domain at the amino-terminal end of downstream Akt, which subsequently changes spatial configuration of Akt and activates 3-phosphoinositide dependent protein kinase $1\left(\mathrm{PDK}_{1}\right)$ [72]. Thirdly, activated $\mathrm{PDK}_{1}$ phosphorylates Thr308 site on catalytic domain of Akt and activated $\mathrm{PDK}_{2}$ (3-phosphoinositide-dependent protein kinase 2) phosphorylates Thr473 site of Akt, which result in activation of Akt at plasma membrane [74]. Finally, activated Akt transmits insulin signal to downstream substrates such as glucose transporter 4 (Glut-4) and glycogen synthase kinase-3 $\beta$ (GSK-3 $\beta$ ), which directly regulate blood sugar levels [73].

At IRS-PI3K-Akt signaling pathway, PI3K belongs to a family of lipid kinases catalyzing inositol and phosphatidylinositol. Meanwhile, PI3K possesses phospholipid kinase and serine/threonine protein kinase activities. It is a heterodimer consisting of regulatory subunit p85 and catalytic subunit p1 10 [75]. Although several factors are able to activate PI3K, its role in insulin signaling pathway is only mediated by insulin. Usually, activated PI3K stimulates catabolism of glucose, anabolism of glycogen, and protein [76]. Akt, a serine/threonine protein kinase, includes $\mathrm{PH}$ domain at amino-terminal end, catalytic and regulatory domains at carboxyl-terminal tail. Two phosphorylation sites, Thr308 and Ser473, are respectively located in catalytic domain 
and regulatory domain serving as target sites of Akt. The activated Akt is responsible for moderating activity of downstream substrate proteins [75]. GSK-3 $\beta$ has a potential for inactivating glycogen synthase (GS); thereby, it has a negative regulatory effect on insulin signal pathway [77]. Glut-4, a carrier protein embedded in the cell, can be phosphorylated by activated Akt. When insulin signal transmits to the cell via insulin signal pathway, intracellular vesicles containing activated Glut- 4 are transferred to the surface of cell membrane, which accelerates the absorption of extracellular glucose (Fig. 2). This mechanism is contributed to abnormity of glucose uptake resulted from disorder of Glut-4 function [76].

Se-polysaccharides possess significant hypoglycemic effect, which mainly attributed to their specific molecular targets for IRS-PI3K-Akt signaling pathway [12]. Ample evidence showed that polysaccharides from Ophiopogon japonicus effectively ameliorated hyperglycemia and insulin resistance of T2DM mice by upregulating important substrate proteins of insulin signal pathway such as $\mathrm{p} 85$ subunit of PI3K, Akt, insulin receptor, InsR, Glut-4, and downregulating GSK-3 $\beta$ [77]. Influence of Se-polysaccharides from Morus alba fruits on high-dose glucoseinduced RIN-m5f pancreatic $\beta$ cells in rat was investigated [12]. And the results indicated that Se-polysaccharides increased glucose consumption by promoting insulin secretion, which was associated with the upregulation of expressions of insulin receptor, InsR and Glut-4 [12]. Based on the abovementioned results, we hypothesize that there are at least three drug targets of Se-polysaccharides on insulin signaling pathway (Fig. 2). Firstly, Se-polysaccharides have the potential for upregulating PI3K expression. Secondly, Se-polysaccharides may upregulate Akt protein in IRS-PI3K-Akt signaling pathway. Put another way, Se-polysaccharides may lead to Akt activation followed by Glut-4 phosphorylation. Thirdly, Se-polysaccharides may inhibit GSK-3 $\beta$ activity and then promote GS activity and then accelerate glycogen synthesis in T2DM mice.

\section{Protective Effect of Se-Polysaccharides Against Oxidative Stress}

Oxidative stress is defined cytologically as a state of imbalance between ROS (e.g., superoxide anion and hydroxyl radicals) and antioxidant defense system (e.g., SOD, CAT, and GSH-Px). Numerous studies revealed that T2DM is strongly linked to an increase in oxidative stress, as supported by ROS-induced damage such as abnormity of insulin signaling, lipid peroxidation, and reduction of antioxidants [58, 78]. Indeed, ROS can activate c-Jun amino-terminal kinase (JNK) in MAPK insulin signaling pathways, and activated JNK may trigger phosphorylation of insulin receptor substrate 1 (InsR-1) [79]. Furthermore, JNK-mediated IRS-1 phosphorylation may exert a dysregulation of some proteins such as PI3K and Glut4, which results in abnormity of blood glucose [80]. The interventions to attenuate oxidative stress may inhibit JNK activity and then decrease blood glucose level and even alleviate insulin resistance. Se-polysaccharides from Ipomoea batatas could remarkably reduce blood glucose level in mice by scavenging free radicals to suppress oxidative stress, which probably attributed to inhibitory activity of JNK [42].

A great number of reports indicated that diabetes mellitus were closely linked to lipid peroxidation as the level of lipid peroxidation in erythrocytes was positively correlated with blood glucose concentrations in diabetic patients [81]. If excessive ROS was not scavenged from cellular environment in time, they might cause peroxidation of polyunsaturated fatty acids. Lipid peroxidation is considered a marker of oxidative damage in T2DM, which would destroy structure, fluidity, and function of cell membrane. Additionally, products of lipid peroxides like MDA may react with some macromolecules (e.g., nucleic acids, lipids, and proteins) and subsequently produce lipofuscins, which are harmful to diabetic patients [82]. Synthetic Se-polysaccharides based on Catathelasma ventricosum could improve activity of antioxidant enzymes such as SOD, CAT, and GSH-Px and reduce MDA in the liver and kidney of STZ-induced diabetic mice [58]. Among antioxidant enzymes, GSH-Px contains $\mathrm{Se}$, whereas SOD does not contain Se, which is a metalloenzyme comprising $\mathrm{Cu} / \mathrm{Zn}-\mathrm{SOD}$, Fe-SOD, Mn-SOD, and NiSOD. Reasons for activating effects of Se-polysaccharides on SOD remain obscure. Much work still need to focus more on effects of Se-polysaccharides on oxidative stress especially lipid peroxidation to clarify the relationship between antioxidant capacity and hypoglycemic activity of Se-polysaccharides, even if Se-polysaccharides can be employed as potential pharmacological interventions and treatment options to prevent or reverse oxidative stress caused by ROS.

\section{Structure-Activity Relationship of Se-Polysaccharides}

Although a huge amount of papers dealt with single biological activity or chemical structure of Se-polysaccharides were published, there are few reports on structure-activity relationship of Se-polysaccharides. It is of theoretical and applied significance to unveil structure-activity relationship of Se-polysaccharides.

\section{Se Content and Hypoglycemic Activity}

In many cases, Se content in Se-polysaccharides is thought to be positively related to hypoglycemic activity of Sepolysaccharides. Yuan et al. [42] confirmed that increasing Se content contributed to higher hypoglycemic activity of synthetic Se-polysaccharides. However, an interesting 
phenomenon is that synthetic Se-polysaccharides based on Artemisia sphaerocephala polysaccharides with Se content of $1703 \mu \mathrm{g} / \mathrm{g}$ did not present superior reducing power when compared to Se-polysaccharides based on Glycyrrhiza uralensis polysaccharides with Se content of $1339 \mu \mathrm{g} / \mathrm{g}$ [32, 33]. Hypoglycemic activity of Se-polysaccharides synthesized via selenite method was not found be positively correlation with their Se content, but the mechanism was still unknown [39]. Thus, whether increasing Se content always augments hypoglycemic activity of Se-polysaccharides still needs further investigation.

\section{Triple-Helical Structure and Hypoglycemic Activity}

Usually, triple-helical structure plays crucial role in hypoglycemic activity of Se-polysaccharides. Liu et al. [52] revealed that hypoglycemic activity of Se-polysaccharides with triplehelical structure was higher than Se-polysaccharides without triple-helical structure. It is noteworthy that triple-helical structure is intimately linked to Se content. Both too high and too low Se contents hampered the formation of triple-helical structure, which led to poor hypoglycemic activity [52].

\section{Molecular Weight and Hypoglycemic Activity}

Gao et al. [57] found that synthetic Se-polysaccharides with a low molecular weight (11.00 kDa) exhibited superior antioxidant activity than native non-Se-polysaccharides with high molecular weight $(15.80 \mathrm{kDa})$. Unfortunately, there is still a lack of direct evidence for the relationship between molecular weight and hypoglycemic activity of Se-polysaccharides.

Some recent studies have revealed the potential impact of micromorphology of Se-polysaccharides on their bioactivities [55]. Monosaccharide composition of Se-polysaccharides derived from Grifola frondosa polysaccharides (non-Se-polysaccharides) was the same as that of non-Se-polysaccharides, while there were significance differences in micromorphology. In marked contrast to non-Se-polysaccharides with branched and entangled chains, Se-polysaccharides were in the shape of irregular fiber with branches of various sizes. Notably, free radical-scavenging ability of Se-polysaccharides was higher than that of non-Se-polysaccharides [55]. More work is needed in this area to clarify effects of micromorphology of Se-polysaccharides on their hypoglycemic activity.

\section{Conclusions and Perspectives}

Natural and artificial forms of Se-polysaccharides frequently exhibit hypoglycemic activity. Presently, numerous researches into chemical structure and hypoglycemic activity of Se-polysaccharides have been carried out, but there are still several challenges in the area. Firstly, future studies should focus more on toxicology of synthetic Sepolysaccharides with high Se content. In most cases, Se content of chemically synthesized Se-polysaccharides is hundreds of times higher than that of natural Se-polysaccharides obtained by bio-transformation. However, potential toxicity of synthetic Se-polysaccharides with high Se content is unknown. Of particular concern is that there is a blurred boundary between the safe dose of synthetic Sepolysaccharides and Se poisoning. For excessive Se intake is associated with skin lesions and nervous system disorder, classical ADME (absorption, distribution, metabolism, and excretion) studies as well as molecular toxicology of synthetic Se-polysaccharides should be conducted. Taking into account the safety of natural Se-polysaccharides, strategies to improve bio-transformation efficiency of natural Se-polysaccharides should be proposed. Secondly, increasing evidence indicated that hypoglycemic activity of Sepolysaccharides is most probably related to IRS-PI3K-Akt signaling pathway. Nevertheless, drug targets and mechanism of action of Se-polysaccharides on insulin signaling pathway are still unclear. Genomics, transcriptomics, proteomics, and metabolomics methodologies should be used to unveil the mechanism underlying hypoglycemic activity of Se-polysaccharides. Thirdly, there are some reports on effects of Se-polysaccharides on diabetes-related enzymes such as $\alpha$-amylase and $\alpha$-glucosidase. However, the structural base of the inhibition of diabetes-related enzymes by Se-polysaccharides still keeps obscure. Fourthly, hypoglycemic activity of Se-polysaccharides is closely related to their chemical structure. Thus, elucidation of structure-activity relationship of Se-polysaccharides is helpful to exploration and utilization of Se-polysaccharides. It is proposed that future research should focus on trying to identify the relationship between hypoglycemic activity and chemical structure of Se-polysaccharides.

Author Contribution Hua-Feng Zhang and Wen-Xia Duan conceived and designed the article. Meng-Yuan Zhang and Jing Feng collected the information and analyzed the data. Wen-Xia Duan and Xiao-Hua Yang wrote the manuscript. Hua-Feng Zhang critically revised the manuscript.

Funding This work was partially funded by Key Research and Development Program of Shaanxi Province (2018ZDXM-SF006/2019FPLS2-01/S2022-YD-QFY-0070), Project of Science and Technology in Shaanxi Province (GJ2021-27/2021XYSF-09), and Science and Technology Development Fund of Macau.

Data availability Data and materials will be made available on reasonable request.

\section{Declarations}

Ethics Approval and Consent to Participate Not applicable. 
Consent for Publication Not applicable.

Competing Interests The authors declare no competing interests.

\section{References}

1. Zhang FR, Feng J, Zhang HF, Zhao AQ, Xue MY, Yang XH (2018) Establishment and application of quantitative analysis method for selenium in Chrysanthemum morifolium. Jiangsu $\mathbf{J}$ Agric Sci 34:927-932

2. Chen L, Huang G (2018) The antiviral activity of polysaccharides and their derivatives. Int J Biol Macromol 115:77-82

3. Zhang J, Saad R, Taylor EW, Rayman MP (2020) Selenium and selenoproteins in viral infection with potential relevance to COVID-19. Redox Biol 37:101715

4. Chen J, Berry MJ (2003) Selenium and selenoproteins in the brain and brain diseases. J Neurochem 86:1-12

5. Rayman MP (2012) Selenium and human health. Lancet 379:1256-1268

6. Gao Z, Zhang C, Liu H, Zhu Y, Ren Z, Jing H, Li S, Zhang J, Liu X, Jia L (2018) The characteristics and antioxidation of Oudemansiella radicata selenium polysaccharides on lipopolysaccharideinduced endo-toxemic mice. Int J Biol Macromol 116:753-764

7. Ru Y, Liu K, Kong X, Li X, Shi X, Chen H (2020) Synthesis of selenylated polysaccharides from Momordica charantia $\mathrm{L}$. and its hypoglycemic activity in streptozotocin-induced diabetic mice. Int J Biol Macromol 152:295-304

8. Zhu J, Yu C, Han Z, Chen Z, Wei X, Wang Y (2020) Comparative analysis of existence form for selenium and structural characteristics in artificial selenium-enriched and synthetic selenized green tea polysaccharides. Int J Biol Macromol 154:1408-1418

9. Sun QL, Dong MM, Wang ZH, Wang CD, Sheng DQ, Li ZH, Huang DB, Yuan CF (2016) Selenium-enriched polysaccharides from Pyracantha fortuneana (Se-PFPs) inhibit the growth and invasive potential of ovarian cancer cells through inhibiting $\beta$-catenin signaling. Oncotarget 7:28369-28383

10 Cheng PG, Phan CW, Sabaratnam V, Abdullah N, Abdulla MA, Kuppusamy UR (2013) Polysaccharides-rich extract of Ganoderma lucidum (M.A. Curtis: Fr.) P. karst accelerates wound healing in streptozotocin-induced diabetic rats. Evid Based Complement Alternat Med 2013:671252

11. Yang Q, Archana V, Kahn BB (2018) Metabolites as regulators of insulin sensitivity and metabolism. Nat Rev Mol Cell Biol 19:654-672

12. Chen C, Zhang B, Fu X, Liu RH (2016) A novel polysaccharide isolated from mulberry fruits (Murus alba L.) and its selenide derivative: structural characterization and biological activities. Food Funct 7:2886-2897

13. Corkey BE (2012) Banting lecture 2011: Hyperinsulinemia: cause or consequence? Diabetes 61:4-13

14. Zhou N, Long HR, Wang CH, Yu L, Zhao MM, Liu XL (2020) Research progress on the biological activities of selenium polysaccharides. Food Funct 11:4834-4852

15. Feng J, Zhang FR, Yang XH, Zhang HF (2019) Phytochemicals in three kinds of Se-enriched compositae flowers and their effects on activity of $\alpha$-glucosidase. Mol Plant Breed 17:2341-2349

16. Zhao ZH, Zhang FR, Li Q, Zhang HF, Wang XY (2018) Antioxidant activities and effects on $\alpha$-amylase and $\alpha$-glucosidase of extract from Se-enriched chrysanthemum morifolium. Nat Prod Res Dev 30:1105-1110

17. Jing YS, Zhang YW, Li JY, Yuan XR, Zhang YG, Wu LF, Zhang DS (2020) Progress of synthesis methods, structural characteristics and biological activities of selenium polysaccharides. Sci Technol Food Ind 42:374-381

18. Pilon-Smits EAH (2019) On the ecology of selenium accumulation in plants. Plants (Basel) 8:197

19. El Mehdawi AF, Jiang Y, Guignardi ZS, Esmat A, Pilon M, PilonSmits EAH, Schiavon M (2018) Influence of sulfate supply on selenium uptake dynamics and expression of sulfate/selenate transporters in selenium hyperaccumulator and nonhyperaccumulator brassicaceae. New Phytol 217:194-205

20. Zhang L, Hu B, Li W, Che R, Deng K, Li H, Yu F, Ling H, Li Y, Chu C (2014) OsPT2, a phosphate transporter, is involved in the active uptake of selenite in rice. New Phytol 201:1183-1191

21. Chen N, Zhao C, Zhang T (2021) Selenium transformation and selenium-rich foods. Food Biosci 40:100875

22. Wang S, Liang D, Wang D, Wei W, Fu D, Lin Z (2012) Selenium fractionation and speciation in agriculture soils and accumulation in corn (Zea mays L.) under field conditions in Shaanxi Province, China. Sci Total Environ 427-428:159-164

23. Hu B, Liang D, Liu J, Lei L, Yu D (2014) Transformation of heavy metal fractions on soil urease and nitrate reductase activities in copper and selenium co-contaminated soil. Ecotoxicol Environ Saf 110:41-48

24. Yuan LJ, Yuan LX, Yi XB, Qin LQ (2016) Physiological function, deficiency and its solutions on selenium: a review. Curr Biotechnol 6:396-405

25. Nancharaiah YV, Lens PN (2015) Selenium biomineralization for biotechnological applications. Trends Biotechnol 33:323-330

26. Zhou X, Wang F, Yang H, Chen J, Ren Y, Yuan Z, Wang X, Wang Y (2014) Selenium-enriched exopolysaccharides produced by Enterobacter cloacae Z0206 alleviate adipose inflammation in diabetic KKAy mice through the AMPK/SirT1 pathway. Mol Med Rep 9:683-688

27. Malinowska E, Krzyczkowski W, Herold F, Łapienis G Ślusarczyk J, Suchocki P, Kuraś M, Turło J (2009) Biosynthesis of selenium-containing polysaccharides with antioxidant activity in liquid culture of Hericium erinaceum. Enzyme Microb Technol 44:334-343

28. Zhang HF, Niu LL, Yang XH, Li L (2014) Analysis of water-soluble polysaccharides in an edible medicinal plant Epimedium: method development, validation and application. J AOAC Int 97:784-790

29. Li HY, Wang YX, Wang C, Zhang SH, Li SH, Zhou GQ, Wang SX, Zhang JC (2017) Extraction, selenylation modification and antitumor activity of the glucan from Castanea mollissima blume. Glycoconj J 34:207-217

30. Böger BR, Salviato A, Valezi DF, Mauro ED, Georgetti SR, Kurozawa LE(2018) Optimization of ultrasound-assisted extraction of grape-seed oil toenhance process yield and minimize free radical formation. J Sci Food Agric98:5019-5026

31. Sun Y, Zhou C, Huang SC, Jiang CJ (2017) Selenium polysaccharide SPMP-2a from Pleurotus geesteranus alleviates $\mathrm{H}_{2} \mathrm{O}_{2}$-induced oxidative damage in $\mathrm{HaCaT}$ cells. Biomed Res Int 2017:4940384

32. Wang JL, Zhao BT, Wang XF, Yao J, Zhang J (2012) Synthesis of selenium-containing polysaccharides and evaluation of antioxidant activity in vitro. Int J Biol Macromol 51:987-991

33. Lian KX, Zhu XQ, Chen J, Liu G, Gu XL (2018) Selenylation modification: enhancement of the antioxidant activity of a Glycyrrhiza uralensis polysaccharide. Glycoconj J 35:243-253

34. Yue CJ, Chen J, Hou RR, Liu J, Li XP, Gao ZZ, Liu C, Wang DY, Lu Y, Li HQ, Hu YL (2015) Effects of selenylation modification on antioxidative activities of Schisandra chinensis polysaccharide. PLoS One 10:e0134363

35. Zhu SY, Hu JH, Liu S, Guo SJ, Jia Y, Li M, Kong WB, Liang JY, Zhang J, Wang JL (2020) Synthesis of Se-polysaccharide mediated by selenium oxychloride: structure features and antiproliferative activity. Carbohydr Polym 246:116545 
36. Xiao XR, Shao ZF, Yu L (2021) A perspective of the engineering applications of carbon-based selenium-containing materials. Chin Chem Lett. https://doi.org/10.1016/j.cclet.2021.03.047

37. Zhao MM, Sun QY, Khogali MK, Liu L, Geng TY, Yu L, Gong DQ (2021) Dietary selenized glucose increases selenium concentration and antioxidant capacity of the liver, oviduct, and spleen in laying hens. Biol Trace Elem Res 199:4746-4752

38. Zhou WJ, Li PZ, Liu J, Yu L (2020) Kilogram-scale production of selenized glucose. Ind Eng Chem Res 59:10763-10767

39. Gao ZZ, Chen J, Qiu SL, Li YY, Wang DY, Liu C, Li XP, Hou RR, Yue CJ, Liu J, Li HQ, Hu YL (2016) Optimization of selenylation modification for garlic polysaccharide based on immuneenhancing activity. Carbohydr Polym 136:560-569

40. Ji YB, Dong F, Lang L, Zhang LW, Miao J, Liu ZF, Jin LN, Hao Y (2012) Optimization of synthesis, characterization and cytotoxic activity of seleno-Capparis spionosa L. polysaccharide. Int J Mol Sci 13:17275-17289

41. Zhao BT, Zhang J, Yao J, Song S, Yin ZX, Gao QY (2013) Selenylation modification can enhance antioxidant activity of Potentilla anserina $\mathrm{L}$. polysaccharide. Int J Biol Macromol 58:320-328

42. Yuan B, Yang XQ, Kou M, Lu CY, Wang YY, Peng J, Chen P, Jiang JH (2017) Selenylation of polysaccharide from the sweet potato and evaluation of antioxidant, antitumor and antidiabetic activities. J Agric Food Chem 65:605-617

43. Zhang HF, Yang XH, Wang Y (2011) Microwave assisted extraction of secondary metabolites from plants: current status and future directions. Trends Food Sci Technol 22:672-688

44. Wang KK (2012) Research progress and application prospects of selenium nanoparticles in livestock production. Feed Res 1:42-44

45. Xiao YD, Huang QL, Zheng ZM, Guan H, Liu SY (2017) Construction of a Cordyceps sinensis exopolysaccharide-conjugated selenium nanoparticles and enhancement of their antioxidant activities. Int J Biol Macromol 99:483-491

46. Chen TF, Wong YS, Zheng WJ, Bai Y, Huang L (2008) Selenium nanoparticles fabricated in Undaria pinnatifida polysaccharide solutions induce mitochondria-mediated apoptosis in A375 human melanoma cells. Colloids Surf B Biointerfaces 67:26-31

47. Zhang JS, Gao XY, Zhang LD, Bao YP (2001) Biological effects of a nano red elemental selenium. BioFactors 15:27-38

48. Estevez H, Garcia-Lidon JC, Luque-Garcia JL, Camara C (2014) Effects of chitosan-stabilized selenium nanoparticles on cell proliferation, apoptosis and cell cycle pattern in HepG2 cells: comparison with other selenospecies. Colloids Surf B Biointerfaces 122:184-193

49. Sasaki T, Takasuka N (1976) Further study of the structure of Lentinan, an anti-tumor polysaccharide from Lentinus edodes. Carbohydr Res 47:99-104

50. Wang YF, Li YF, Liu YY, Chen XQ, Wei XL (2015) Extraction, characterization and antioxidant activities of Se-enriched tea polysaccharides. Int J Biol Macromol 77:76-84

51. Liu YT, Sun J, Rao SQ, Su YJ, Li JH, Li CM, Xu SD, Yang YJ (2013) Antidiabetic activity of mycelia selenium-polysaccharide from Catathelasma ventricosum in STZ-induced diabetic mice. Food Chem Toxicol 62:285-291

52. Liu YT, You YX, Li YW, Zhang L, Yin LL, Shen YB, Li C, Chen H, Chen SJ, Hu B (2017) The characterization, selenylation and antidiabetic activity of mycelial polysaccharides from Catathelasma ventricosum. Carbohydr Polym 174:72-81

53. Sheng Y, Liu GC, Wang ML, Lv ZY, Du PD (2017) A selenium polysaccharide from Platycodon grandiflorum rescues PC12 cell death caused by $\mathrm{H}_{2} \mathrm{O}_{2}$ via inhibiting oxidative stress. Int J Biol Macromol 104:393-399

54. Gu YD, Qiu Y, Wei X, Li Z, Hu ZQ, Gu YY, Zhao YZ, Wang YD, Yue TL, Yuan YH (2020) Characterization of selenium-containing polysaccharides isolated from selenium-enriched tea and its bioactivities. Food Chem 316:126371
55. Li Q, Wang W, Zhu Y, Chen Y, Zhang WJ, Yu P, Mao GH, Zhao T, Feng WW, Yang LQ, Wu XY (2016) Structural elucidation and antioxidant activity of a novel Se-polysaccharide from Seenriched Grifola frondosa. Carbohydr Polym 161:42-52

56. Miu JL, Li GY, Wang B (2000) Study on the physical and chemical properties and the molecular structure of Seleno-chitosan. Chin J Mar Drugs 19:7-10

57. Gao PY, Bian J, Xu SS, Liu CF, Sun YQ, Zhang GL, Li DQ, Liu XG (2020) Structural features, selenization modification, antioxidant and anti-tumor effects of polysaccharides from alfalfa roots. Int J Biol Macromol 149:207-214

58. Liu YT, Zeng SQ, Liu YX, Wu WJ, Shen YB, Zhang L, Li C, Chen H, Liu AP, Shen L, Hu B, Wang CL (2018) Synthesis and antidiabetic activity of selenium nanoparticles in the presence of polysaccharides from Catathelasma ventricosum. Int J Biol Macromol 114:632-639

59. Wang L, Wang GY, Zhang JJ, Zhang GQ, Jia L, Liu XN, Deng P, Fan KM (2011) Extraction optimization and antioxidant activity of intracellular selenium polysaccharide by Cordyceps sinensis SU-02. Carbohydr Polym 86:1745-1750

60. Zhu ZY, Liu F, Gao H, Sun HQ, Meng M, Zhang YM (2016) Synthesis, characterization and antioxidant activity of selenium polysaccharide from Cordyceps militaris. Int J Biol Macromol 93:1090-1099

61. Moller DE (2001) New drug targets for type 2 diabetes and the metabolic syndrome. Nature 414:821-821

62. Adib M, Peytam F, Rahmanian-Jazi M, Mahernia S, Bijanzadeh HR, Jahani M, Mohammadi-Khanaposhtani M, Imanparast S, Faramarzi MA, Mahdavi M (2018) New 6-amino-pyrido [2,3d] pyrimidine-2,4-diones as novel agents to treat type 2 diabetes: a simple and efficient synthesis, alpha-glucosidase inhibition, molecular modeling and kinetic study. Eur J Med Chem 155:353-363

63. Zhai Y, You YM, Ren XP (2015) Mechanism of action and major adverse effects of diabetes drugs. Strait Pharm J 27:194-197

64. Vidyasagar K, Chandrasekar B, Chhabra M, Bhansali A, Bansal D (2020) Efficacy and safety of commonly used insulin analogues in the treatment of diabetic ketoacidosis: a bayesian indirect treatment comparison. Clin Ther 42:e115-e139

65. Sharma AK, Taneja G, Kumar A, Sahu M, Sharma G, Kumar A, Sardana S, Deep A (2019) Insulin analogs: glimpse on contemporary facts and future prospective. Life Sci 219:90-99

66. Kim KY, Nam KA, Kurihara H, Kim SM (2008) Potent alphaglucosidase inhibitors purified from the red alga Grateloupia elliptica. Phytochemistry 69:2820-2825

67. Tadera K, Minami YJ, Takamatsu K, Matsuoka T (2005) Inhibition of glucosidase and amylase by flavonoids. J Nutr Sci Vitaminol 52:149-153

68. Zhang H, Li DS, Tan KX, Xiang JQ, Liu W (2018) Effects of selenium polysaccharide from Cyclocarya paliurus (batal) ijinskaja on $\alpha$-glucosidase and glucose consumption in HepG2 Cells. Sci Technol Food Ind 2:46-49+56

69. Liu YT, Zeng SQ, Tang QQ, You YX, Li YW, Chen D, Liu AP, Li C (2016) Preparation of Se-enriched polysaccharides from Catathelasma ventricosum and ananlysis of antioxidant and antihyperglycemic activities. Mod Food Sci Technol 32:60-65

70. Nielsen JE, Borchert TV (2000) Protein engineering of bacterial $\alpha$-amylases. Biochim Biophys Acta 1543:253-274

71. Holman GD, Kasuga M (1997) From receptor to transporter:insulin signalling to glucose transport. Diabetologia 40:991-1003

72. Song ZL, Wu LL, Qin LL, Sun W, Qin TY, Pan YJ, Liu TH (2019) Advances in the PI3K/AKt signaling pathway and diabetes mellitus. Modern Tradit Chin Med Mater Med 21:1264-1268

73. Lai YT, Ge L, Huang PP, Wang GD, Jiang XY, Pang ST, Xie $\mathrm{Y}$, Lin AW (2021) Advances in the role of PI3K/AKt signaling 
pathway in gestational diabetes mellitus. Mater Child Health Care China 36:234-237

74. Cantrell DA (2001) Phosphoinositide 3-kinase signalling pathways. J Cell Sci 114:1439-1445

75. Dai YX, Li YC, Liang FR (2018) Advances in the PI3K/AKt/ GSK3 $\beta$ pathway affecting glucose metabolism and cognitive processes. Cardiovasc Dis J Integr Tradit Chin West Med 6:28-29

76. Kido Y, Nakae J, Accili D (2001) The insulin receptor and its cellular targets. J Clin Endocrinol Metab 86:972-979

77. Wang LY, Yuan W, Xu DS, Ruan KF, Feng L, Wang S (2012) MDG-1, a polysaccharide from Ophiopogon japonicus exerts hypoglycemic effects through the PI3K/AKt pathway in a diabetic KKAy mouse model. J Ethnopharmacol 143:347-354

78. Jiang S, Wang YH, Ren DY, Li JR, Yuan GG, An LP, Du PG, Ma J (2014) Antidiabetic mechanism of Coptis chinensis polysaccharide through its antioxidant property involving the JNK Pathway. Pharm Biol 53:1022-1029

79. Hotamisligil GS (2006) Inflammation and metabolic disorders. Nature 444:860-867
80. Kaneto H, Matsuoka T, Katakami N, Kawamori D, Miyatsuka T, Yoshiuchi K, Yasuda T, Sakamoto K, Yamasaki Y, Matsuhisa M (2007) Oxidative stress and the JNK pathway are involved in the development of type 1 and type 2 diabetes. Curr Mol Med 7:674-686

81. Styskal J, Remmen HV, Richardson A, Salmon AB (2012) Oxidative stress and diabetes: what can we learn about insulin resistance from antioxidant mutant mouse models? Free Radic Biol Med 52:46-58

82. Li DD, Fang L, Li J, Gao RH (2015) Study on relation of hba1c and anti-oxidant level, lipid peroxidation status in patients with type 2 diabetes mellitus. J Community Med 13:15-17

Publisher's Note Springer Nature remains neutral with regard to jurisdictional claims in published maps and institutional affiliations. 